\title{
Carbon optimism
}

\author{
The use of diamond, graphene and carbon nanotubes is becoming increasingly common in photonic \\ applications, and several recent notable achievements suggest that carbon has a bright future in photonics.
}

Historically, compound semiconductors, glasses and polymers have all proved to be important materials for constructing a wide range of photonic devices such as light sources, detectors, waveguides and modulators, to name but a few. It now seems that carbon may also have an increasingly important role in future photonic devices.

In particular, the common allotropes of carbon - diamond, graphene (a single layer of graphite just one atom thick) and the carbon nanotube - are attracting significant attention from photonics researchers in both academia and industry. Indeed, in both the News \& Views and Research Highlights sections of this issue of Nature Photonics, we report on several recent research findings from carbon photonics in the optical world.

First, consider diamond. Although it is most famous for its aesthetics, incredible hardness, thermal conductivity and strength, it also has useful optical properties ${ }^{1}$. It is well known that the crystal lattice of diamond can feature an intrinsic point defect, in which two carbon atoms are replaced by a nitrogen atom and a lattice vacancy.

These defects are commonly referred to as nitrogen-vacancy centres, and belong to a family of 'colour centres' that take their name from the fact that they give diamond a colour (a perfect diamond free from such defects is colourless). In particular, when optically pumped, an isolated nitrogen-vacancy centre emits single photons of visible light, making diamond a promising solid-state roomtemperature light source for applications in quantum information processing. Indeed, Quantum Communications Victoria - a spin-off company from the University of Melbourne in Australia - is now selling commercial single-photon sources based on tiny diamond crystals grown directly on the tips of optical fibres.

Diamond single-photon sources also featured on the front cover of the March issue of our sister journal Nature Nanotechnology (pictured), in which researchers from the US and Germany reported an efficient diamond nanowire single-photon source based on an array of nanopillars in diamond ${ }^{2}(\sim 200 \mathrm{~nm}$ in diameter and $2 \mu \mathrm{m}$ high). Each nanopillar features a single nitrogen-vacancy centre and, thanks to its nanowire waveguiding geometry, offers a much-improved input coupling of green pump light and output

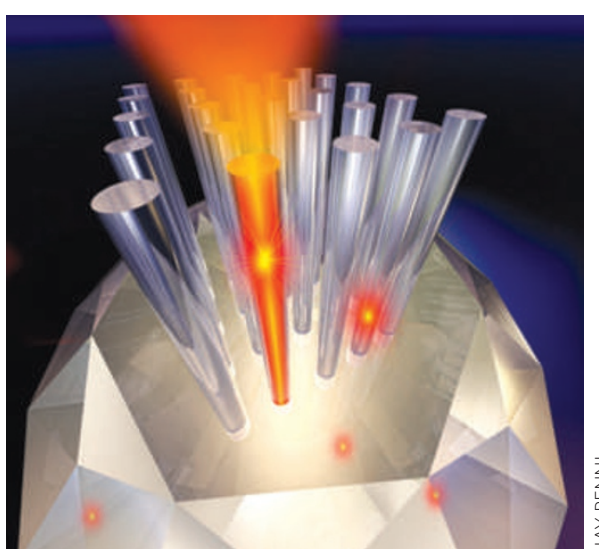

may turn out to be a valuable method for allowing new regimes of optical operation, as described on page 200 . The surrounding carbon nanotubes effectively modify the local electric field environment of the dye, allowing classical rules that govern radiative transitions, such as Kasha's rule, to be broken.

The optical properties of graphene and carbon nanotubes ${ }^{4}$ are certainly a topic of great interest to the US firm IBM, whose researchers at the Thomas J. Watson Research Center have been active in investigating the potential for constructing optoelectronic devices such as nanotube LEDs and graphene photodetectors ${ }^{5}$, with much recent success. The aim is to create nanoscale devices with unique optical properties and integrate them with semiconductor electronics.

IBM has recently reported a $\mathrm{p}-\mathrm{n}$ junction coupling of red single photons, compared with that of bulk diamond. The result is a source that the researchers claim is ten times more efficient than bulk diamond.

As well as having applications in quantum optics, diamond may also be behind a new high-power solid-state laser source of yellow light, described on page 202. Such a source is desirable because scientists usually rely on dye lasers for producing such wavelengths. However, as recently reported in Optics Letters ${ }^{3}$, researchers have now developed a diamond laser that uses the Raman effect - a vibrational-induced downshift in the frequency of light emission - to convert several watts of green $(532 \mathrm{~nm})$ pump laser light into short pulses of yellow $(573 \mathrm{~nm})$ light, with a conversion efficiency of around $30 \%$. Diamond is an attractive material for making such a laser because its high thermal conductivity prevents thermal problems when operating at high powers, and its naturally large Raman shift neatly bridges the gap between yellow wavelengths and green wavelengths, in which high-power sources are readily available (for example, frequency-doubled Nd:YAG lasers). Such a source of intense yellow pulses could prove useful for applications in biomedicine, especially for the treatment of skin stains.

Carbon is also proving useful in its other forms, particularly as graphene and carbon nanotubes. Both have been shown to be useful as saturable absorbers, allowing a convenient means of passively modelocking a laser and thus generating short pulses. Furthermore, filling hollow carbon nanotubes with suitable light-emitting dyes single-walled carbon-nanotube-based LED with a light generation efficiency that is three orders of magnitude greater than previous devices $^{6,7}$. In addition, the company's latest detector results are detailed in a Letter that has just gone live on our website using our Advance Online Publication service ${ }^{8}$. The researchers report a new design for a fast and responsive graphene photodetector that is compatible with $10 \mathrm{Gbit} \mathrm{s}^{-1}$ datastreams, suggesting that carbon does indeed have a promising future for use in telecommunications networks. The big attraction of graphene in particular is that it features an incredibly broad absorption spectrum that spans from the ultraviolet to the mid-infrared. This could, in principle, be used to greatly broaden the $1.5 \mu \mathrm{m}$ spectral transmission window of opticalfibre communications and expand it to both longer and shorter wavelengths, allowing multiple-wavelength channel communication on a far larger scale than is being used today. 